International scientific and technical conference

DOI: $10.34185 / 1991-7848 . i t m m .2020 .01 .012$

\title{
ДИНАМИКА ПРОКАТКИ СВАРНЫХ ШВОВ
}

Веренев В.В., д.Т.н., ст.н.с., Подобедов Н.И., к.т.н., ст.н.с.

\section{Институт черной металлургии им. 3.И. Некрасова НАН Украины, Украина}

Аннотация. Представлены результаты исследований переходных процессов и нагрузок в межклетевых промежутках при холодной непрерывной прокатке полос со сварными соединениями. Применена разработанная ранее и адаптированная к 5-клетевому стану математическая модель и компьютерная программа заполнения полосой непрерывной группы клетей, записанная в абсолютных значениях переменных. Рассмотрены два вида возмущений, действующих со стороны шва: его утолщение на 0,1-0,2 мм длиной до 20 мм и превышение сопротивления деформации на 10-20 \% по сравнению с стыкуемыми полосами. При входе утолщенного шва в клеть № 1 происходит кратковременное снижение (ослабление) заднего натяжения до 0,6 начального значения между клетями № 1 и 2. Действие шва в следующих клетях существенно ослабляется ввиду трансформации его формы. Более динамичный случай наступает при увеличенном сопротивлении деформации участка шва: формируются рывки переднего и заднего натяжений до 1,5-2,2 установившегося значения, увеличивается их зависимость от скорости прокатки. В обоих случаях на шве остаются отпечатки вертикальных колебаний клетей, шов становится разнотолщинным. Непосредственно после шва наблюдается утонение («пережим») полосы на 0,07 мм, что способствует нежелательной концентрации продольных напряжений.

Ключевые слова: НЕПРЕРЫВНАЯ ПРОКАТКА, СВАРНЫЕ СОЕДИНЕНИЯ, МЕЖКЛЕТЕВЫЕ НАТЯЖЕНИЯ, СКОРОСТЬ, ОПЕРЕЖЕНИЕ, ТОЛЩИНА ПОЛОСЫ.

Введение. На непрерывных полосовых станах холодной прокатки наблюдаются обрывы полос, сваренных встык. Как показывает анализ публикаций, кроме качества сварки полос и собственно швов выделяется другая причина обрывов. Она состоит в том, что шов как возмущающий фактор во время прокатки вызывает существенные динамические нагрузки в системе «прокатная клеть - очаг деформации - полоса - линия привода валков». 
Ministry of Education and Science of Ukraine

The National Metallurgical Academy of Ukraine, Dnipro, 17 - 19 March, 2020

Изучению данной стороны вопроса наиболее полно посвящены работы $[1,2]$, однако, по нашему мнению, исследования далеки от завершения. Задача данной работы состоит в том, чтобы полнее раскрыть характер протекания переходных процессов в полосе, очаге деформации и оборудовании во время последовательной прокатки сварного соединения в непрерывной группе клетей.

Основной материал. Для решения задачи разработанную математическую модель динамики заполнения непрерывной шестиклетевой группы горячей полосой, представленную в абсолютных значениях переменных [3], адаптировали к непрерывному пятиклетевому стану холодной прокатки 1700. В модели учтены упругие колебания клети и линии привода валков, уравнения межклетевых натяжений и транспортного переноса с запаздыванием толщины полосы между клетями. Основное внимание уделялось исследованию межклетевых натяжений в взаимосвязи с толщиной шва и околошовного участка полосы.

Рассмотрены следующие варианты возмущений со стороны сварного стыка.

А. Участок сварного шва длиной 20 мм изначально превышает на 0,1-0,2 мм толщину стыкуемых полос (условно геометрическое возмущение).

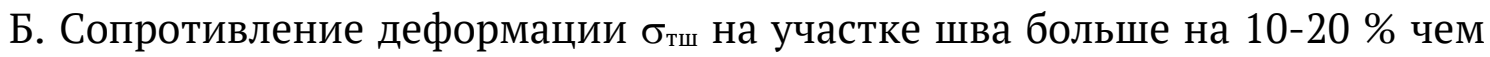
стыкуемых полос $\sigma_{\text {тш }}>\sigma_{\text {тп }}$ при равной толщине шва и полос. В. Сочетание возмущений по п. А и Б.

Получены следующие результаты моделирования и закономерности.

А. Геометрическое возмущение. 1. Возмущение со стороны шва, как геометрического фактора, в наибольшей мере действует во второй клети, к последним клетям заметно ослабляется и не приводит к существенным рывкам натяжения в последних промежутках.

2. При прохождении шва через очаг деформации заднее натяжение колеблется: сначала происходит его ослабление до амплитуды $(0,6-0,8) \cdot \mathrm{T}_{\text {уст. }}$

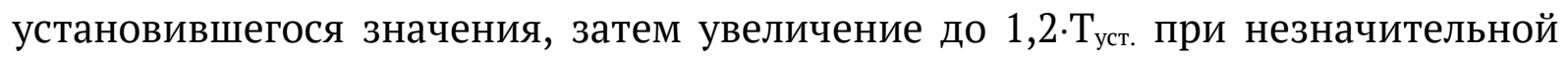
амплитуде колебаний переднего натяжения. 
International scientific and technical conference Information Technologies in Metallurgy and Machine building - ITMM 2020

3. По мере прохождения клетей абсолютное и относительное превышение толщины шва уменьшаются согласно выравнивающим свойствам клетей.

4. После каждой клети не сохраняется ровной поверхность шва, его толщина колеблется в соответствии с частотой собственных колебаний упругой системы клети.

5. Непосредственно на шве или сразу за ним появляются кратковременные утонения (пережимы) до 0,08 мм. От клети к клети эти утонения повторяются в тех же самых местах, что может привести к их наложению и усилению концентрации продольных напряжений.

Б. Силовое возмущение. 1. При прокатке шва с увеличенным сопротивлением деформации всегда образуется рывок заднего натяжения в 1,3-2 раза больше, чем при увеличенной толщине.

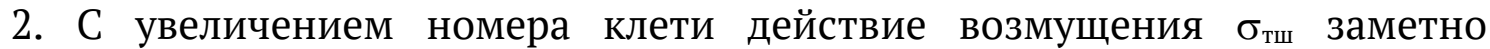
возрастает, рывки натяжения к последним промежуткам, где полоса становится тоньше, увеличиваются.

3. C увеличением скорости прокатки рывки натяжений при прохождении участка шва через клети возрастают сильнее, чем в варианте А, и достигают $2,2 \cdot \mathrm{T}_{\text {уст. }}$.

4. Как и в случае геометрического возмущения происходит аналогичная трансформация поверхности и толщины шва и околошовного участка, с возможным наложением утонений.

В. Одновременное действие возмущений. 1. В переходном процессе по натяжениям в первых двух клетях сначала сказывается превышение толщины

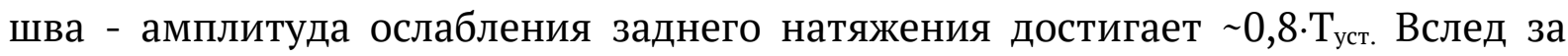
этим наступает существенный рывок заднего натяжения до $(2-2,3) \cdot \mathrm{T}_{\text {уст. и }}$ переднего до 1,5·Т уст. Эти пики зависят от скорости прокатки - увеличиваются.

2. Увеличивается амплитуда колебаний толщины шва примерно в 2 раза в обе стороны с возможностью совмещения пережимов в одном месте.

3. После клетей № 1 и 2 превышение толщины шва, как возмущения, существенно ослабевает, а действие свойств металла шва возрастает вместе со скоростью прокатки и уменьшением толщины прокатываемой полосы. Таким образом, совместное влияние на динамику межклетевых натяжений и 
Ministry of Education and Science of Ukraine

The National Metallurgical Academy of Ukraine, Dnipro, 17 - 19 March, 2020

толщины шва и полосы двух возмущений - толщины и свойств металла шва проявляется в первых двух клетях. В остальных клетях сказывается влияние только сопротивления деформации металла шва.

Графики получаемых с помощью модели переходных процессов таких параметров, как скорость входа и выхода полосы из валков, опережения металла в очаге деформации, усилия прокатки соответствуют всем закономерностям теории прокатки.

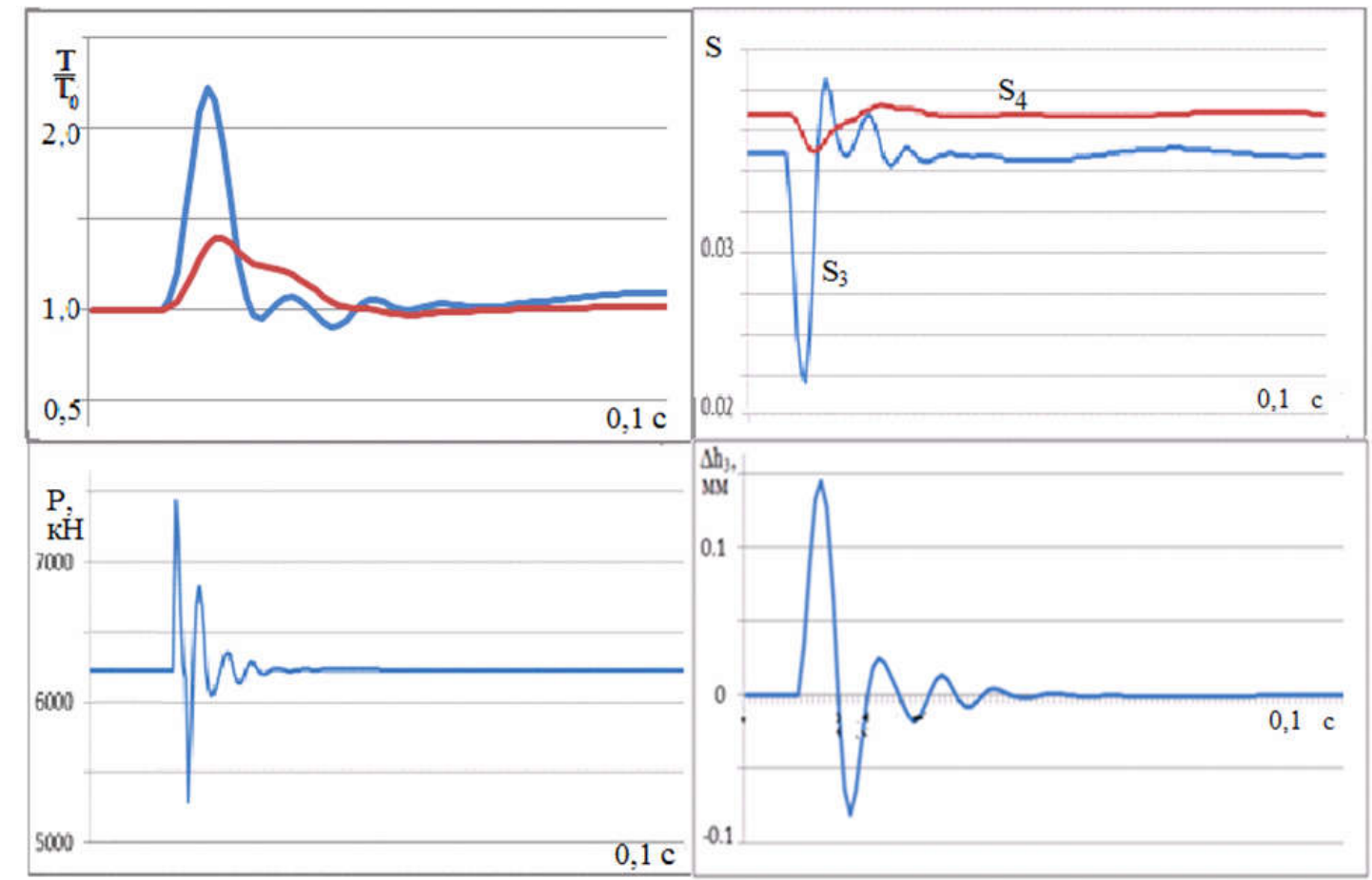

Рисунок - Переходные процессы при прохождении шва с

повышенным сопротивлением деформации через клеть № 3: 1 и 2 -

относительное заднее и переднее натяжение, $\mathrm{S}_{3}, \mathrm{~S}_{4}$ - опережение в клетях

№ 3 и 4, Р и $\Delta \mathrm{h}$ - усилие прокатки и отклонение толщины шва

Разработанная модель позволяет выбрать рациональное сочетание режима обжатий и скорости прокатки при заданных параметрах сварного соединения и клетей стана по критерию наименьших рывков межклетевых натяжений и отклонению толщины шва и полосы и за счет этого повысить прокатываемость сварных соединений. Компьютерная программа может быть использована в режиме сопровождения непрерывной прокатки в случаях отклонений в подготовке сварных соединений. Скоростью прокатки швов можно регулировать и предупреждать большие рывки натяжений, если предварительно определять фактическое значение сопротивления

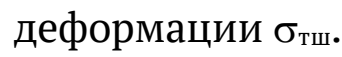


International scientific and technical conference

Information Technologies in Metallurgy and Machine building - ITMM 2020

Выводы. 1. Сварные швы как возмущение, действующее со стороны прокатываемой полосы, вызывают существенные рывки межклетевых натяжений. При этом увеличенная толщина шва влияет на динамику в первых клетях, а увеличенное значение сопротивления деформации зоны шва сильнее проявляется в рывках натяжений к последним клетям. На форму шва по толщине решающее влияние оказывают колебания упругой системы клетей, вызывая нежелательные утонения участка полосы вслед за швом.

\section{Литература}

1. Прокатка металла со сварными соединениями. В.Л. Мазур, В.И. Мелешко, Д.П. Галкин [и др.] - М.: Металлургия, 1985. - 112 с.

2. Мазур В.Л., Ноговицын А.В. Теория и технология листовой прокатки. (Численный анализ и технические приложения). Днепропетровск, РИА «Днепр-VAL». 2010. - 498 с.

3. Веренев В.В., Путноки А.Ю., Подобедов Н.И. Переходные процессы при непрерывной прокатке. Днепр: Літограф. 2017. - 116 с.

\section{DYNAMICS OF ROLLING OF WELDED JOINTS}

Verenev Valentyn, Podobedov Mykolai

Annotation. The results of studies of transients and loads in the inter-cage spaces during continuous cold rolling of strips with welded joints are presented. A mathematical model developed earlier and adapted to a 5-stand mill and a computer program for filling a continuous group of stands with a strip recorded in absolute values of variables are applied. Two types of perturbations acting from the side of the seam are considered: its thickening by 0.1-0.2 $\mathrm{mm}$ up to $20 \mathrm{~mm}$ long and the excess of deformation resistance by $10+20 \%$ compared to abutting strips. When a thickened seam enters cdet No. 1, a short-term decrease (weakening) of the back tension occurs to 0.6 of the initial value between stands No. 1 and 2 . The action of the seam in the following stands is significantly weakened due to the transformation of its shape. A more dynamic case occurs when the deformation resistance of the weld metal is exceeded: jerks of the front and rear tension are formed up to 1.5-2.2 steady-state values, their dependence on the rolling speed increases. In both cases, imprints of vertical cage vibrations remain on the seam, and the seam becomes multi-thickness. Thinning ("pinch”) of the strip by $0.07 \mathrm{~mm}$ is observed immediately 
Ministry of Education and Science of Ukraine

The National Metallurgical Academy of Ukraine, Dnipro, 17 - 19 March, 2020

after the seam, which contributes to an undesirable concentration of longitudinal stresses.

Key words: CONTINUOUS ROLLING, WELDED JOINTS, INTERSTAND TENSION, SPEED, LEAD, STRIP THICKNESS.

\section{References}

1. Rolling metal with welded joints. V.L. Mazur, V.I. Meleshko, D.P. Galkin [et al.] - M .: Metallurgy, 1985. - 112 p.

2. Mazur V.L., Nogovitsyn A.V. Theory and technology of sheet rolling. (Numerical analysis and technical applications). Dnepropetrovsk, RIA Dnepr-VAL. 2010.- 498 p.

3. Verenev VV, Putnoki A.Yu., Podobedov N.I. Transients during continuous rolling. Dnipro, Lithograph, 2017. - $116 \mathrm{~s}$. 